\title{
Selecting Weights for Different Type Measurements in WLS State Estimation
}

\author{
Yubin Yao, Zhiliang Wu and Dan Wang \\ School of Marine Engineering \\ Dalian Maritime University \\ Dalian, China \\ yaoyubin@dlmu.edu.cn
}

\begin{abstract}
Power system state estimation provides accurate and complete data for several online advanced application software in energy management systems. Its main consideration is how to acquire accurate data. State estimation are usually solved by weighted least squares estimation, and selecting a reasonable weight for every measurement is the most important means to achieve a good estimation. Traditionally variance of measurement is selected as its weight. But measurements of different type have different effect on the accuracy of state estimation even if they have same error. Selection of weights must take the measurement type into account, so a method of selecting weights for measurements of different type is presented. Firstly accuracy index of state estimation is defined as the sum of the squares of errors of every measurement; then accuracy index of state estimation for every measurement is calculated by perturbation method; finally mean accuracy index of state estimation of measurements of each type is calculated and used to design a coordination factor to adjust weights for measurements of different type. IEEE30 network is analyzed by the proposed method, and the results prove the effectiveness of the proposed method.
\end{abstract}

Keywords- State estimation; weighted least squares estimation; weight; measurement type; coordination factor; perturbation method; accuracy index

\section{INTRODUCTION}

To provide accurate and complete data for several online power system studies such as dispatcher power flow, security analysis, state estimation plays a very important role in the monitoring and control of power systems. It turns the raw network data which is less accurate and incomplete into good data which is accurate and complete. Several methods have been presented since state estimation was introduced in power systems [1-5]. Among them, the predominant approach is the weighted least squares (WLS) method [1-10].

The main consideration of state estimation is the degree of accuracy of its output data. The best way to acquire accurate data is solving the problem by the weighted least squares method. Factors influenced the accuracy of state estimation have been investigated, and many methods to improve the accuracy of state estimation have been presented. Ref. [6] analyzed effect of instrument transformer accuracy class on the WLS state estimator accuracy. Ref. [7] included voltage measurements in branch current state estimation for distribution systems to improve accuracy of state estimation. After comparing two main types of WLS state estimators for distribution systems which based on node voltage state and branchcurrent state respectively, Ref. [8] concluded that branchcurrent estimators with the slack bus voltage as one of the state variables can give estimation accuracies comparable to the voltage ones.

Selecting a reasonable weight for every measurement is the key to achieve higher accurate estimate with given measurements for weighted least squares estimation. Since measurement errors have a Gaussian distribution, it is good choice to use the reciprocal of measurement error variance as weight for the measurement. However measurement error varies due to variation of operating conditions of telecommunication systems and aging of the instruments, the weights need to be continuously updated [9-10]. Ref. [9] presented a novel algorithm to estimate and adaptively update measurement variances by estimating measurement variances from sensitivity matrix which depicts the relationship between the measurement variances and the covariance of their residuals. Ref. [10] presented a simple method to estimate measurement error variance based on the sample variances of the measurement residuals calculated in historical records, so time-consuming calculation of the sensitivity matrix is averted.

The reciprocals of measurement error variances are usually chosen as weights of measurements without considering the type of measurements. For measurements of different type, same measurement error has different influence on the accuracy of state estimation. So this paper presents a new way to select weights for measurements with their types being considered. Firstly this paper defines accuracy index of state estimation to evaluate the degree of accuracy of state estimation; then investigates the effect of errors of different type measurements on the accuracy of state estimation according to this index via perturbation method; finally designs a coordination factor to adjust weights for measurements of different type. IEEE30 network is studied by the proposed method, and the results prove the effectiveness of the proposed method.

\section{FUNDAMENTAL EQUATIONS OF STATE ESTIMATION}

The purpose of state estimation is to acquire state variables by measurements. Due to inaccuracy of measurement equipments and noise of telecommunication system, there is error between the measurement and its true value as follows

$$
\mathbf{z}=\mathbf{z}_{0}+\mathbf{e}
$$


where $\mathbf{z}$ is measurement vector of dimension $m, \mathbf{z}_{0}$ is true value vector of dimension $m$, e is $m$-dimensional measurement error vector with zero mean.

In fact true value is unknown in a real system, so it is substituted by its estimate value and gives the relationship of state variables and measurements as follows

$$
\mathbf{z}=\mathbf{h}(\mathbf{x})+\mathbf{r}
$$

where $\mathbf{x}$ is state vector of dimension $n$ consisted of voltage magnitudes and phase angles, $\mathbf{h}(\mathbf{x})$ is $m$-dimensional function vector of state vector represents the desired estimation of $\mathbf{z}, \mathbf{r}$ is $m$-dimensional measurement residual vector with zero mean.

The measurement errors are commonly assumed to have a Gaussian distribution with unknown parameters. The estimate vector $\hat{\mathbf{x}}$ can be solved by weighted least squares (WLS) method.

The objective function of the WLS method is as follow

$$
J=[\mathbf{z}-\mathbf{h}(\hat{\mathbf{x}})]^{\mathrm{T}} \mathbf{W}[\mathbf{z}-\mathbf{h}(\hat{\mathbf{x}})]
$$

where $\mathbf{W}$ is the weighting matrix of dimension $m \times m$, the superscript $(\mathrm{T})$ denotes transposition of the matrix.

The weighting matrix is diagonal matrix, its element $w_{i i}$ is as follows

$$
w_{i i}=1 / \sigma_{i}^{2}
$$

where $\sigma_{i}^{2}$ is measurement error variance of the $i$ th measurement.

The function $\mathbf{h}(\hat{\mathbf{x}})$ in (3) is nonlinear function about $\hat{\mathbf{x}}$ which makes the objective function being nonlinear one. To solve this nonlinear objective function, linearization of function $\mathbf{h}(\hat{\mathbf{x}})$ is first step, linearization of function $\mathbf{h}(\hat{\mathbf{x}})$ is as follows

$$
\mathbf{h}(\hat{\mathbf{x}})=\mathbf{H} \hat{\mathbf{x}}
$$

where $\mathbf{H}=\partial \mathbf{h}(\hat{\mathbf{x}}) / \partial \hat{\mathbf{x}}$ is Jacobian matrix of dimension $m$ $\times n$ at a given value of the vector $\hat{\mathbf{x}}$.

To minimize (3) and take into account (5) give the normal equation as follows

$$
\mathbf{H}^{\mathrm{T}} \mathbf{W H} \Delta \hat{\mathbf{x}}=\mathbf{H}^{\mathrm{T}} \mathbf{W}[\mathbf{z}-\mathbf{h}(\hat{\mathbf{x}})]
$$

To solve (6) for $\Delta \hat{\mathbf{x}}$ and update the estimate $\hat{\mathbf{x}}$, and this procedure is repeated for several times until $\Delta \hat{\mathbf{x}}$ satisfies the given tolerance.

\section{THE Proposed Method to ChOOSE WeIGHTS FOR MEASUREMENTS OF DIFFERENT TYPE}

As a special case of the general problem of estimation, the problem of power system state estimation has its specificity. While measurements for the general problem of estimation are of same type, measurements for the problem of power system state estimation may be of different types. Measurements for power system state estimation can be classified as: bus power injections, line (branch) power flows, bus voltage magnitudes, line (branch) current magnitudes. By the usage of measurements, power system state estimation can be classified as many algorithms. Among these algorithms, the injections only algorithm or the line only algorithm use measurements of only one type, others use measurements of two or more types. If measurements of more than one type are used in power system state estimation algorithms, the effect of errors of different type measurements on accuracy of state estimation should be investigated.

\section{A. Accuracy Index of State Estimation}

To evaluate accuracy of state estimation, we define a accuracy index $J_{a}$ as follows

$$
J_{a}=\left[\mathbf{z}_{0}-\mathbf{h}(\hat{\mathbf{x}})\right]^{\mathrm{T}}\left[\mathbf{z}_{0}-\mathbf{h}(\hat{\mathbf{x}})\right]
$$

where $\mathbf{z}_{0}$ is true value vector of dimension $m, \mathbf{h}(\hat{\mathbf{x}})$ is the desired estimation of $\mathbf{z}$ calculated by estimated state variable vector $\hat{\mathbf{x}}$.

Since the true value vector $\mathbf{z}_{0}$ in (7) is unknown for a real power system, we can use the basic power flow data to evaluate accuracy of state estimation by this accuracy index.

In the real power system, we can evaluate accuracy index of state estimation under a given operating condition via output data of power flow. These results include branch power flows, bus power injections, and bus voltage magnitudes. The results of power flow are regarded as the true values, and the results plus small errors as measurements. Then state estimation is carried out by using these data, and accuracy index can be calculated to evaluate the influence of the error of every measurement on accuracy of state estimation.

\section{B. Calculation of Accuracy Index of State Estimation for \\ Each Measurement}

For measurements of different type, the effect of the same error on the accuracy of state estimation is different. The effect of error of each measurement on the accuracy of state estimation can be investigated by perturbation method. The perturbation method is widely applied in power system analysis such as economic dispatch, optimal operation, security assessment [11-13].

By perturbation method, we set a small error $e_{i}$ to a appointed measurement $z_{i}$, others are error-free measurements. By WLS state estimation, we can obtain estimated state variables and estimated measurements, and then the accuracy index of state estimation for each measurement.

\section{Choice of Weights}

After the accuracy index of state estimation for every measurement has been computed, we can calculate mean accuracy index for measurements of each type as $J_{a T 1}, J_{a T 2}$, $J_{\text {aT3 }}$ which are mean accuracy index of state estimation for voltage magnitude measurements, power injection measurements, branch power flow measurements respectively.

To coordinate measurements of different type, coordination factor is designed.

Set the coordination factor $k_{w i}$ for bus voltage measurement $z_{i}$ as 1.0 . 
The coordination factor $k_{w i}$ for bus power injection measurement $z_{i}$ as follows

$$
k_{w i}=J_{a T 1} / J_{a T 2}
$$

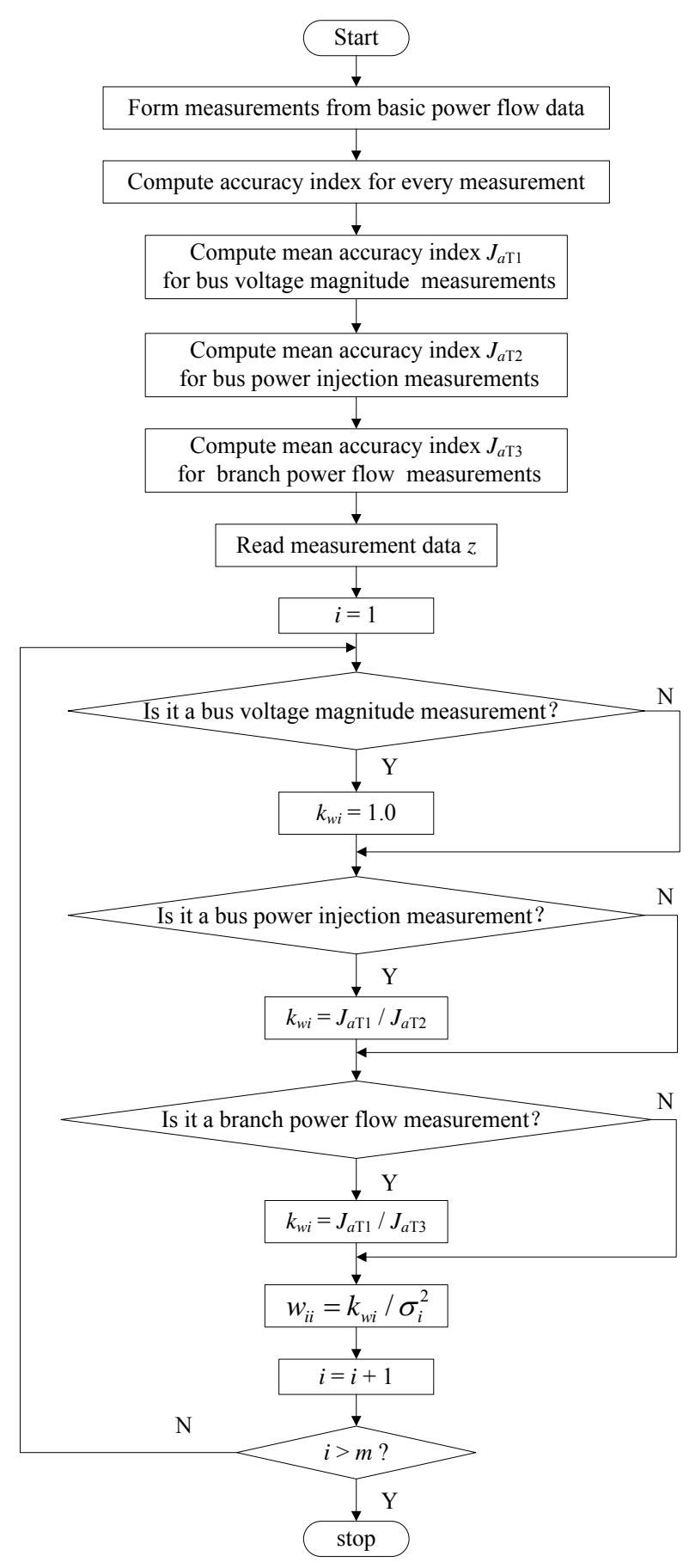

Figure 1. Flow chat of setting weights for measurements

The coordination factor $k_{w i}$ for branch power flow measurement $z_{i}$ as follows

$$
k_{w i}=J_{a T 1} / J_{a T 3}
$$

With the coordination factor we set weights for measurements as follows

$$
w_{i i}=k_{w i} / \sigma_{i}^{2}
$$

The flow chart of setting weights for measurements is shown as Fig .1. The procedure includes three steps: calculating accuracy index of state estimation for all measurements by perturbation method; calculating mean accuracy index of state estimation for measurements of each type; calculating coordination factor for measurements of each type; set weight for every measurement.

\section{CASE STUdY}

IEEE30 system is used to testify our conclusions. There are 30 buses, 41 branches (include transmission lines and transformers), 6 power sources, 2 shunt capacitors. There are 164 branch power flow measurements, 60 bus injection measurements and 30 bus voltage magnitude measurements. All electrical quantities are in per unit. The one-line diagram of IEEE30 system is shown in Fig. 2 .

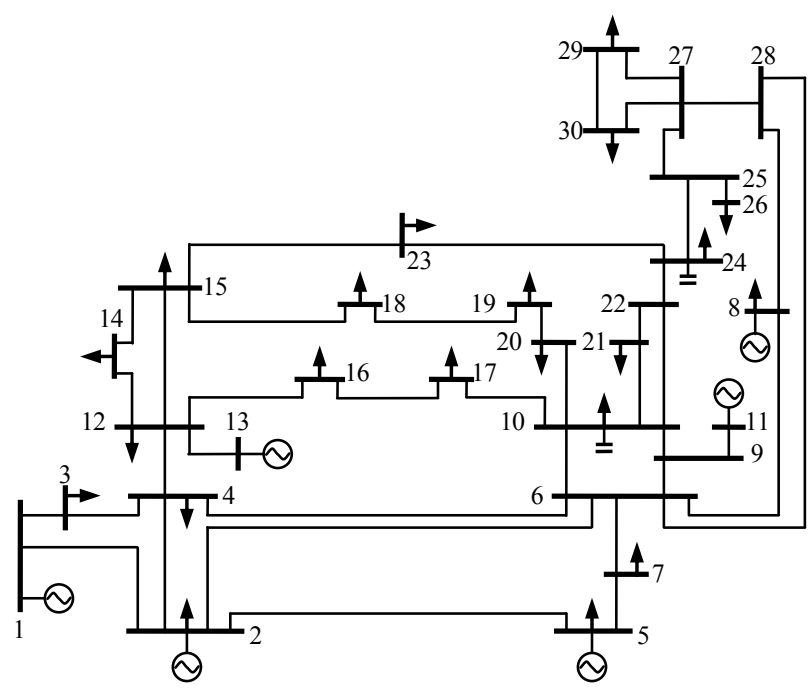

Figure 2. IEEE30 system

\section{A. Mean Accuracy Index of State Estimation for Measurements of Each Type}

The accuracy index of state estimation is calculated for every measurement by perturbation method, the perturbation quantity is $1 \%$ of the measurement being evaluated. The mean accuracy indexes of state estimation for measurements of each type are calculated and list in Table I. 
TABLE I. MEAN ACCURACy IndeXes of StATE Estimation FOR MEASUREMENTS OF EACH TYPE

\begin{tabular}{cc}
\hline Measurement Type & Mean sensitivity Index \\
\hline Bus voltage magnitudes & $5.07217 \times 10^{-6}$ \\
Bus power injections & $2.68729 \times 10^{-6}$ \\
Branch power flows & $7.08390 \times 10^{-7}$ \\
\hline
\end{tabular}

According the mean accuracy indexes of state estimation for measurements of each type listed in Table I, the coordination factors are calculated as 1.0, 1.9, 7.2 for bus voltage magnitude measurements, bus power injection measurements and branch power flow measurements respectively.

\section{B. Accuracy Index for Different Methods}

The test system is carried out by traditional method and the proposed method respectively for five cases:

Case 1: The error of each measurement is $1 \%$.

Case 2: The error of each measurement is $2 \%$.

Case 3: The error of each measurement is 3\%.

Case 4: The error of each measurement is $4 \%$.

Case 5: The error of each measurement is 5\%.

The accuracy index by different methods is listed in Table II. For the traditional method, weight of every measurement is 1.0; for the presented method, weights of bus voltage magnitude measurements are 1.0, weights of bus power injection measurements and weights of branch power flow measurements are 1.9 and 7.2 respectively.

TABLE II. COMPARISON OF ACCURACY INDEX BY DIFFERENT METHODS

\begin{tabular}{cccc}
\hline \multirow{2}{*}{ Cases } & \multicolumn{2}{c}{ Accuracy Index } & \\
\cline { 2 - 3 } & $\begin{array}{c}\text { Traditional } \\
\text { method }\end{array}$ & $\begin{array}{c}\text { Proposed } \\
\text { method }\end{array}$ & \\
\hline case 1 & 0.0039892 & 0.0038559 & $3.34 \%$ \\
case 2 & 0.0159652 & 0.0153602 & $3.79 \%$ \\
case 3 & 0.0359287 & 0.0344922 & $4.00 \%$ \\
case 4 & 0.0638805 & 0.0612304 & $4.15 \%$ \\
case 5 & 0.0998211 & 0.0955537 & $4.28 \%$ \\
\hline
\end{tabular}

As showed in Table II, the presented method is more accurate than traditional method, and improves the accuracy more when the errors are big.

\section{CONCLUSION}

Measurements of different type have different effect on the accuracy of state estimation even if they have same error. The degree of the influence can be evaluated by accuracy index of state estimation of each measurement with same error. To coordinate the different influence of same error of measurements of different type, a coordination factor which is according to the mean accuracy index of state estimation of measurements of each type is introduced in weight setting for measurements. Setting weight for measurements considering measurement type can improve the accuracy of state estimation.

\section{ACKNOWLEDGMENT}

This work was supported in part by NSFC under Grant 61273137 to Dan Wang.

\section{REFERENCES}

[1] Ali Abur and Antonio Gómez Expósito. Power system state estimation. New York: Marcel Dekker, Inc. 2004.

[2] D. P. Kothari and I. J. Nagrath. Modern power system analysis. 3ed. New York: McGraw-Hill Companies, Inc. 2003.

[3] L. Holten, A. Gjelsvik, S. Aam, F.F. Wu and W.H.E Liu. "Comparison of different methods for state estimation," IEEE Trans. on Power Systems, vol. 3, no. 4, Dec. 1988, pp. 1798-1806.

[4] S. Sarri, M. Paolone, R. Cherkaoui, A. Borghetti, F. Napolitano and C. A. Nucci. "State estimation of active distribution networks: comparison between WLS and iterated kalman-filter algorithm integrating PMUs," proc IEEE PES Innovative Smart Grid Technologies Europe, Oct. 2012, pp. 1-8.

[5] Catalina Gómez-Quiles, Antonio de la Villa Jaén and Antonio Gómez-Expósito. "A factorized approach to WLS state estimation," IEEE Trans. on Power Systems, vol. 26, no. 3, August. 2011, pp. 1724-1732.

[6] Markos Asprou, Elias Kyriakides and Mihaela Albu. "The effect of instrument transformer accuracy class on the WLS state estimato accuracy," proc IEEE Power and Energy Society General Meeting July 2013, pp.1-5

[7] M. Baran, J. Jung and T. McDermott. "Including voltage measurements in branch current state estimation for distribution systems," proc IEEE Power Energy Society General Meeting(PES 09), July 2009, pp.1-5

[8] M. Pau, P. A. Pegoraro and S. Sulis. "WLS distribution system state estimator based on voltages or branch-currents: accuracy and performance comparison," proc IEEE Instrumentation and Measurement Technology Conference, May 2013, pp.1-6.

[9] Guangyi Liu, Erking Yu and Y. H. song. "Novel algorithms to estimate and adaptively update measurement error variance using power system state estimation results," Electric Power System Research, vol. 47, no. 1, Oct. 1998, pp. 57-64.

[10] Shan Zhong and Ali Abur. "Auto tuning of measurement weights in WLS state estimation," IEEE Trans. on Power Systems, vol. 19, no. 4, Nov. 2004, pp. 2006-2013.

[11] T. T. Nguyen and A. Karimishad. "Fast and accurate method for dynamic security-constrained economic dispatch via sensitivity analysis," proc IEEE Power Engineering Society General Meeting June 2007, pp. 1-8.

[12] E. A. Belati, E. C. Baptista and G. R. M. Da Costa. "Optimal operation studies of the power system via sensitivity analysis,' Electric Power Systems Research, Vol. 75, no. 1, July 2005, pp. 79-84

[13] A. T. Sarić and A. M. Stanković. "Model uncertainty in security assessment of power systems," IEEE Trans. on Power Systems Vol. 20, no. 3, Aug. 2005, pp. 1398-1407. 